\title{
PedsQL-4 Psychosocial Health Summary Score
}

National Cancer Institute

\section{Source}

National Cancer Institute. PedsQL-4 Psychosocial Health Summary Score. NCI

Thesaurus. Code C121529.

The mean value computed as the sum of the score of individual items over the number of items answered in the Emotional, Social, and School Functioning Scales. 